\title{
FORMULAÇÕES COM ALIMENTOS CONVENCIONAIS PARA NUTRIÇÃO ENTERAL OU ORAL ${ }^{1}$
}

\author{
Edma M. ARAÚJO2, Hilary C. MENEZES ${ }^{2, *}$
}

\begin{abstract}
RESUMO
A nutrição enteral domiciliar é um tratamento econômico e seguro usado na prevenção da desnutrição e manutenção da qualidade de vida. Nesse estudo, foi analisada a formulação FCM (CARVALHO et al. 1992) e foram desenvolvidas formulações (F1 e F2) para obter quantidades e qualidade dos componentes conforme literatura. As fórmulas foram desenvolvidas com alimentos convencionais de baixo custo, factíveis de preparo em domicílio, disponíveis em regiões menos desenvolvidas, onde as dietas industrializadas não são comercializadas. Foram determinadas propriedades como viscosidade, osmolalidade, gotejamento e valor nutricional. As fórmulas $\mathrm{F} 1 \mathrm{e}$ F2 apresentaram menor viscosidade e distribuição calórica (proteína $14 \%$, gordura $33 \%$, carboidrato $53 \%$ ), fibra alimentar (8,16 g/2 L) e densidade calórica ( $1 \mathrm{kcal} / \mathrm{mL}$ ) melhores que FCM (proteína 19\%, gordura 33\%, carboidrato 48\%, fibra alimentar 4,68 g/2 L e densidade calórica $0,87 \mathrm{kcal}$ ). As fórmulas F1 e F2 apresentaram soluções mais fluidas que FCM, gotejamento de $60-80$ gotas/minuto. As osmolalidades ficaram isotônicas. A adição de fubá de milho, Soymilk ${ }^{\circledast}$ e Nidex ${ }^{\circledR}$ melhoraram o valor nutritivo e a fluidez de F1 e F2, viabilizando a administração de maior quantidade em menor tempo. As formulações F1 e F2 são opções para uma individualização de dieta enteral normal por gastrostomia em terapia domiciliar.
\end{abstract}

Palavras-chave: dietas, suplemento oral, valor nutritivo, tratamento domiciliar, desnutrição.

\section{SUMMARY}

FORMULATIONS FOR ENTERAL OR ORAL NUTRITION USING CONVENTIONAL FOODS. Home enteral nutrition is a safe, economic treatment used in the prevention of malnutrition and in the maintenance of life quality. In this study the formulation FCM (CARVALHO et al., 1992) was analysed, and the formulations F1 and F2 developed, aiming at obtaining the component quantities and qualities cited in the literature. The formulations were developed using low cost conventional foods, easy to prepare domestically and available in less developed regions where industrialised diets are not commercialised. The viscosity, osmolality, dripping properties and nutritive value were determined. The F1 and F2 formulations presented lower viscosities than FCM and improved caloric distribution ( $14 \%$ protein, $33 \%$ fat, $53 \%$ carbohydrate), food fibre content $(8.16 \mathrm{~g} / 2 \mathrm{~L}$ ) and caloric density ( $1 \mathrm{kcal} / \mathrm{mL})$ than FCM ( $19 \%$ protein, $33 \%$ fat, $48 \%$ carbohydrate, caloric density of $0.87 \mathrm{kcal} / \mathrm{mL}$ and food fibre content of $4.68 \mathrm{~g} / 2 \mathrm{~L}$ ). The formulations $\mathrm{F} 1$ and F2 were more fluid than FCM, dripped at 60-80 drips/minute and showed isotonic osmolalities. The addition of corn grits, Soymilk ${ }^{\circledR}$ and Nidex ${ }^{\circledast}$ improved the nutritive value and flow properties, making it possible to administer more in less time. The formulations represent options to individualise normal enteral diets in home therapy.

Keywords: diets, oral supplements, nutritive value, home care, malnutrition.

\section{1- INTRODUÇÃO}

A Nutrição enteral (NE) domiciliar é um tratamento econômico e seguro, pois elimina gastos com internação, evita a contaminação hospitalar e viabiliza o convívio de pacientes com familiares.

A NE é de importância fundamental para prevenir e tratar as deficiências de macronutrientes e melhorar a recuperação do paciente, fornecendo a quantidade de nutrientes compatíveis com o metabolismo existente [8].

Nos países desenvolvidos, é comum o uso de dietas enterais industrializadas. No Brasil, o consumo desse tipo de dieta vem aumentando gradativamente. As dietas industrializadas são práticas, nutricionalmente completas e oferecem maior segurança quanto ao controle microbiológico e composição centesimal. No entanto, não estão acessíveis para a maioria da

$\overline{{ }^{1} \text { Recebido para publicação em 24/1/2005. Aceito para publicação em }}$ 6/7/2006 (001471)

${ }^{2}$ Núcleo de Estudos e Pesquisas em Alimentação (NEPA/UNICAMP),

Rua Albert Einstein, 291, CEP 13083-970, Campinas (SP), Brasil,

E-mail:nepa@unicamp.br

* A quem a correspondência deve ser enviada população brasileira. A estimativa é de que cerca de $50 \%$ dos hospitais brasileiros não trabalhem com dietas industrializadas e, possivelmente, outros $20 \%$ as adquiram em casos selecionados, não fazendo parte da rotina do hospital [5]. Uma avaliação preliminar permite estimar que 30\% dos hospitais trabalham com dietas industrializadas e $70 \%$ não [5]. Embora fórmulas industrializadas e prontas para uso estejam disponíveis há mais de 20 anos, muitas instituições preferem usar as dietas enterais preparadas na unidade hospitalar, por razões econômicas e culturais [18, 25].

Nesse estudo, foram desenvolvidas duas formulações (F1 e F2) para NE ou oral, factíveis de preparo em domicílio, poliméricas, isentas de lactose, para pacientes com dieta normal e função gastrintestinal digestiva e absortiva íntegras.

\section{2 - MATERIAL E MÉTODOS}

\section{1 - Matéria-prima}

Para compor as dietas, foram escolhidos alimentos convencionais de baixo custo, disponíveis em regiôes menos desenvolvidas e distantes dos grandes centros comerciais 
onde as NE industrializadas não são comercializadas. Foram utilizados: carne bovina (coxão mole sem gordura e sem aponevrose), arroz agulhinha tipo-1, açúcar refinado, fubá de milho amarelo tipo mimoso, ovo vermelho tamanho médio, chicória (Cichorium endivia L.), cenoura (Daucus carota L.), óleo de soja, sal iodado, Soymilk ${ }^{\circledR}$ sabor natural - extrato de soja em pó desengordurado, açúcar, óleo de soja refinado, maltodextrina, vitaminas (A, B1, B2, B6, B12, C, D, E, K, niacina, ácido pantotênico, ácido fólico e biotina), minerais (cálcio, ferro, fósforo, magnésio, zinco, cobre, iodo, sódio e manganês), aroma natural de baunilha e goma guar como espessante - e Nidex ${ }^{\circledR}$ (maltodextrina). Todos os alimentos (Tabela 1) foram adquiridos em supermercados de Campinas/SP.

\section{2 - Métodos}

\subsection{1 - Formulação das dietas}

A fórmula FC [6] foi usada como básica e foi desenvolvida uma versão modificada (FCM) (Tabela 1) mais diluída com quantidades menores de ingredientes, adequadas para um volume de $2 \mathrm{~L}$ (Tabela 1 ). Foram desenvolvidas as formulações F1 e F2 (Tabela 1) com outros alimentos, para obter quantidades e qualidade em concordância com os padrões da literatura [1, $1124,27,29]$. Os alimentos foram pesados em balança analítica (Scientech SA 120, Tecnal $^{\circledR}$, Piracicaba, Brasil).

TABELA 1 - Alimentos, formulações e quantidades - 2 L.

\begin{tabular}{|c|c|c|c|c|}
\hline \multicolumn{5}{|c|}{ Formulações } \\
\hline Alimentos (g) & $\mathrm{FC}^{1}$ & $\mathrm{FCM}^{2}$ & $F 1^{3}$ & $\mathbf{F}^{3}$ \\
\hline Arroz & 128 & 110 & - & - \\
\hline Açúcar refinado & 120 & 105 & - & - \\
\hline Fubá de milho & - & - & 80 & 80 \\
\hline Nidex ${ }^{\circledR}$ & - & - & 185 & 185 \\
\hline Carne & 400 & 348 & 200 & 200 \\
\hline Ovo & - & - & 50 & - \\
\hline Soymilk $^{\circledR}$ & - & - & 70 & 75 \\
\hline Cenoura & 160 & 140 & 50 & 50 \\
\hline Chicória & - & - & 100 & 100 \\
\hline Óleo de soja & 48 & 42 & 40 & 45 \\
\hline Sal iodado & - & - & 3 & 4 \\
\hline
\end{tabular}

${ }^{1} \mathrm{FC}$ [5]; ${ }^{2} \mathrm{FCM}$ modificada [5]; e ${ }^{3} \mathrm{~F} 1$ e ${ }^{3} \mathrm{~F} 2$ formuladas no NEPA.

\subsection{2 - Determinação do conteúdo nutricional e energético}

Para calcular o valor nutricional das dietas, foram usadas tabelas de composição de alimentos [4, 7, 10, 12, 20], sódio [9] e informação nutricional da embalagem (Olvebra Industrial S. A) para Soymilk ${ }^{\circledR}$ e (Nestlé Brasil Ltda) para Nidex $^{\circledR}$. Com base na literatura, foi estabelecido um teor de sólidos de $20 \%$ [6], densidade calórica de $1 \mathrm{kcal} / \mathrm{mL}$ [11, 24], volume de 2 L e valor calórico total de 2000 kcal. Foi con- siderado equilíbrio dos macronutrientes, com distribuição calórica percentual para proteína de, no máximo, 20\% [11], lipídios de 30-35\% [1, 29] e carboidratos de 50-60\% [1, 29]. Foram calculadas as kcal não protéicas/g de nitrogênio [27], fator 6,25 [22] e carga de soluto renal $(\mathrm{CSR}=(\mathrm{Na}+$ $\mathrm{K}+\mathrm{Cl}(\mathrm{mEq} / \mathrm{L})+$ proteína $\mathrm{x}$ fator 5,7$)[27]$.

O teor de água das formulações foi calculado subtraindo-se os sólidos do volume total.

\subsection{3 - Preparo das formulações}

No preparo das dietas, foi considerada a importância de se obter soluções com partículas pequenas e leves, possíveis de escoar pelo capilar e boa qualidade microbiológica.

As dietas foram preparadas em panela de pressão (12 psi, cap. 4 L, Colck ${ }^{\circledR}$, Brasil). A carne e a cenoura foram cortadas em pedaços pequenos, colocadas em água fria e levadas à cocção em fogo baixo durante $1 \mathrm{~h}$ e 40 min. Depois, foram acrescentados o fubá, a chicória e o sal às formulações F1 e F2, e o arroz pré-cozido à fórmula FCM. Após 15 min de fervura, foi adicionado o ovo (F1) sob agitação, sendo a mistura mantida em ebulição durante mais 15 min. Em seguida, a panela foi retirada do fogo e resfriada em água fria até temperatura ambiente. A preparação foi então triturada e homogeneizada em liquidificador (Pic-Liq, Arno ${ }^{\circledR}$, São Paulo, Brasil) junto com o Nidex ${ }^{\circledR} \mathrm{e}$ o Soymilk ${ }^{\circledR}$, peneirada (tela de nylon plana, abertura de $1 \mathrm{~mm}$ de diâmetro), colocada em recipiente de vidro e mantida em refrigerador $\left(5^{\circ} \mathrm{C}\right)$ até o dia seguinte para a realização das determinações.

\subsection{4 - Determinações físicas e físico-químicas}

$\mathrm{O}$ pH foi determinado em amostras de $12 \mathrm{~mL}$ (à temperatura de $23{ }^{\circ} \mathrm{C}$ e sem adição de óleo) usando um pHmetro (Tecnal ${ }^{\circledR}-2$, Piracicaba, Brasil).

A osmolalidade foi determinada usando-se um osmômetro (Advanced ${ }^{\circledast}$ Wide-Range 3WII, Norwood, Inglaterra). As amostras foram congeladas, centrifugadas (Sorval ${ }^{\circledR}, \mathrm{RC}-5 \mathrm{C}$, USA) durante $5 \mathrm{~min}$ (2000 rpm) e alíquotas de $250 \mu \mathrm{L}$, em duplicata, foram separadas do sobrenadante (16 mL) para a leitura direta da osmolalidade.

A viscosidade aparente (Pascal. s) foi determinada pelo método rotacional [23] em reômetro (Rheocontroller, RC $20 \mathrm{~N}$, Haake $^{\circledR}$, Alemanha) de placas paralelas e rotativas, usando-se amostras de $0,8 \mathrm{~mL}$.

Para observação da estabilidade e da homogeneidade, as soluções foram preparadas, acondicionadas em béquer de vidro, colocadas em refrigerador durante $24 \mathrm{~h}$ e observadas por inspeção visual [28].

O gotejamento (gotas/min) foi realizado pelo método gravitacional, usando-se $200 \mathrm{~mL}$ de dieta em frascos plásticos $\left(\right.$ Darrow $^{\circledR}$ ) com capacidade $600 \mathrm{~mL}$ e equipo (Laboratório B. Braun ${ }^{\circledR}$ ) para administração de soluções. 


\section{3 - RESULTADOS E DISCUSSÃO}

A distribuição do valor calórico total (VCT) das formulações é mostrada na Figura 1. Comparando-se os resultados com os da literatura, nota-se que todas as formulações estão adequadas para $\mathrm{NE}$ ou oral [1, 11] sendo que a formulação FCM apresentou um teor de proteína mais elevado, próximo de $20 \%$ do VCT, caracterizando-a como hiperprotéica [1, 11]. Os teores de gordura ficaram com as mesmas proporções para todas as formulações [1]. A proporção de carboidratos ficou mais baixa para a formulação FCM e em melhores proporções para as formulações F1 e F2 [1]. A distribuição calórica ficou em proporções mais equilibradas para as fórmulas F1 e F2, caracterizando-as como normoprotéicas, normoglicídicas e normolipídicas [1, 11] podendo, assim, ser uma opção para fornecer macronutrientes para paciente em terapia nutricional domiciliar num tipo de alimentação que apresenta boa tolerância da maioria dos pacientes assistidos por uma equipe especializada [2, 21, 25].

No entanto, as formulações podem apresentar variação em relação ao valor nutricional devido à imprecisão dos dados disponíveis nas tabelas de composição dos alimentos.

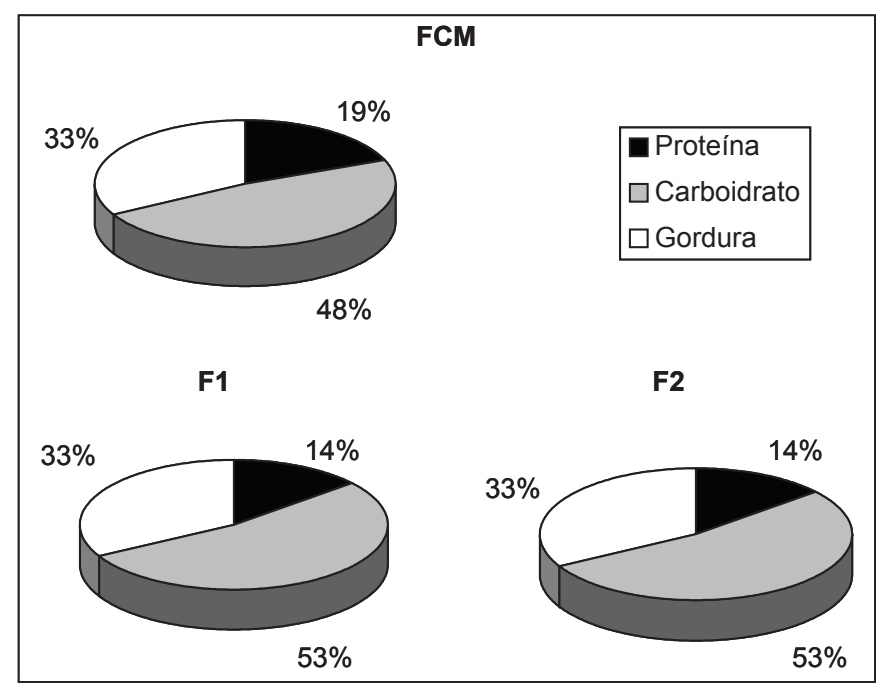

FIGURA 1 - Distribuição do valor calórico total das formulações FCM [5], F1 e F2 formuladas no NEPA - 2 L.

Na Tabela 2, estão relacionadas as principais propriedades das formulações. Os resultados demonstram que as proporções de alimentos utilizadas nas formulações F1 e F2 foram adequadas para se obter uma densidade calórica de $1 \mathrm{kcal} / \mathrm{mL}$, conforme referências especificas [1, 11], padrão geralmente utilizado nas formulações industriais. Já para a formulação FCM, as proporções resultaram numa solução menos fluida, de densidade calórica de $0,87 \mathrm{kcal} / \mathrm{mL}$.

A proporção de kcal não protéica por grama de nitrogênio das formulações F1 e F2 ficou adequada [1], sendo mais indicada para pacientes com aporte protéico normal, enquanto a formulação FCM, com proporção mais baixa, ficou mais indicada para pacientes com aporte protéico maior.

Os percentuais de adequação de fibra alimentar, em relação ao valor calórico de $2000 \mathrm{kcal}$ [15], ficaram próximos de $32,64 \%$ (F1 e F2) e 18,72\% (FCM). As recomendações nutricionais para fibra alimentar são de $25 \mathrm{~g} /$ dia para uma dieta de $2000 \mathrm{kcal}$ [17], porém, estudo recente se refere à opção de suplementação da DE com um preparado básico de fibras, em pequena quantidade, em caráter preventivo [26].

As formulações FCM e F2, sem ovo, apresentaram teores de colesterol abaixo dos recomendados [13, 29], e a formulação F1, com ovo, teor equivalente [13, 29] (Tabela 2).

As soluções ficaram homogêneas, com aspecto uniforme, não ocorrendo separação de fases após $24 \mathrm{~h}$ de preparo. A concentração de sólidos possibilitou uma estabilidade dos sistemas dispersos permitindo um escoamento das soluções através do equipo, sem agitação, durante os testes de gotejamento. A proporção recomendada [6] de sólidos é de $20 \%$. Para as fórmulas F1 e F2, obtivemos valores acima de $20 \%$ e soluções mais fluidas quando comparadas com a fórmula FCM, cuja concentração de sólidos é de 17,97\% (Tabela 2).

O gotejamento médio para as formulações $\mathrm{F} 1$ e F2, que apresentaram menor viscosidade que FCM no escoamento pelo capilar e nos testes reológicos (Figura 2), foi de 60 a 80 gotas por minuto, com uma variação de mais ou menos 10 gotas. Apesar das dietas com alimentos convencionais apresentarem viscosidade mais elevada [19] que as dietas industrializadas, esses volumes obtidos para as formulações F1 e F2 ficaram adequados [27] para administração por gotejamento gravitacional, podendo ser obtida uma vazão menor no período de adaptação da dieta, com aumento gradativo. A formulação FCM mostrou-se mais densa que F1 e F2 (Figura 2) e, no escoamento pelo capilar, devido à formação de gel em função da presença de amido no arroz [14] após hidratação, aquecimento e resfriamento [15, 16], o número máximo de gotas por minuto foi de 22 a 26 (Tabela 2), com controle do equipo liberado, não sendo possível obter variações de vazão.

As osmolalidades ficaram isotônicas para as três formulações quando comparadas com a classificação da literatura [11,24], característica das fórmulas enterais poliméricas compostas de alimentos intactos (Tabela 2), sendo que os valores mais elevados foram das formulações preparadas com adição de sal iodado, um elemento químico de efeito osmótico por conter o eletrólito sódio com propriedade de dissociação em partículas menores [1], porém necessário para a adequação da quantidade diária de iodo. As dietas isotônicas não causam desconforto abdominal, podendo ser administrado um volume maior em menos tempo, de acordo com a tolerância digestiva do paciente.

As preparações apresentaram sabor e cheiro agradável, levemente adocicado e cor creme. 
TABELA 2 - Descrição das propriedades físico-químicas das formulações $-2 \mathrm{~L}$.

\begin{tabular}{|c|c|c|c|}
\hline \multicolumn{4}{|c|}{ Formulações } \\
\hline Propriedades & $\mathrm{FCM}^{1}$ & $\mathrm{~F}^{2}{ }^{2}$ & $\mathrm{~F}^{2}{ }^{2}$ \\
\hline Valor calórico total (kcal) & 1743 & 2065 & 2052 \\
\hline $\begin{array}{l}\text { Densidade calórica } \\
(\mathrm{kcal} / \mathrm{mL})\end{array}$ & 0,87 & 1,03 & 1,02 \\
\hline Sólidos (\%) & 17,97 & 22,12 & 21,74 \\
\hline Água (mL) & 1640,68 & 1558,28 & 1560,56 \\
\hline $\begin{array}{l}\text { Kcal não proteíca/g de } \\
\text { nitrogênio (Kcal/g N) }\end{array}$ & $104: 1$ & $149: 1$ & $159: 1$ \\
\hline Colesterol (mg/2 L) & 205,32 & 310,50 & 118,00 \\
\hline Fibras alimentares (g) & 4,68 & 8,16 & 8,16 \\
\hline $\begin{array}{l}\text { Osmolalidade } \\
\left(\mathrm{mOsm} / \mathrm{Kg} \mathrm{H} \mathrm{H}_{2} \mathrm{O}\right)\end{array}$ & 276 & 330 & 344 \\
\hline $\mathrm{Na}(\mathrm{mEq} / \mathrm{L})$ & 5,47 & 31,86 & 38,90 \\
\hline $\mathrm{K}(\mathrm{mEq} / \mathrm{L})$ & 20,81 & 26,07 & 26,06 \\
\hline $\mathrm{Cl}(\mathrm{mEq} / \mathrm{L})$ & 3,75 & 29,90 & 37,32 \\
\hline $\begin{array}{l}\text { Carga soluto renal } \\
(\mathrm{mOsm} / 2 \mathrm{~L})\end{array}$ & 540,46 & 617,20 & 616,20 \\
\hline $\mathrm{pH}$ & 5,80 & 6,09 & 6,01 \\
\hline $\begin{array}{l}\text { Gotejamento gravitacional } \\
\text { (gotas/minuto) }\end{array}$ & 22 a 26 & 60 a 80 & 60 a 80 \\
\hline
\end{tabular}

Para vitaminas e minerais, os percentuais de adequação, em relação à ingestão diária recomendada para adultos (IDR) [3], estão relacionados nas Tabelas 3 e 4 . Esses dados representam os teores de minerais e vitaminas dos alimentos in natura e dos alimentos industrializados que foram adicionados às dietas após o preparo, devendo ser consideradas as perdas no processo de cocção dos alimentos in natura. Como, no Brasil, não há tabela de composição de alimentos que ofereça dados precisos e completos, a necessidade de suplementação desses elementos deve ser considerada para adequação das necessidades diárias individuais. De acordo com os resultados obtidos no cálculo com tabelas de composição de alimentos, a maioria das vitaminas e minerais das formulações F1 e F2 (Tabelas 3 e 4) ficaram adequadas às recomendações IDR [3] com exceção dos minerais Mg, Mn, Se e das vitaminas B1, B2, C, E, B6 e ácido fólico para a formulação $\mathrm{F} 1$ e dos minerais $\mathrm{Mg}$, Se, $\mathrm{Cu}, \mathrm{Mn}, \mathrm{Ca}$ e das vitaminas B1, B2, C, E e ácido fólico para a formulação F2. A maioria dos minerais e vitaminas da fórmula FCM (Tabela 3 e 4) ficaram com percentuais abaixo das recomendações IDR [3].

As quantidades de sal adicionadas na composição das formulações F1 (3 g) e F2 (4 g) foram suficientes para suprir as necessidades mínimas diárias de iodo e manter os teores adequados de sódio nas dietas [13].

O melhor tempo de preparo encontrado para cocção dos alimentos foi de duas horas e dez minutos. Com esse tempo, testado várias vezes, os alimentos ficaram bem cozidos, e foi possível obter um aproveitamento total dos nutrientes.

Na Figura 2, tem-se as curvas reológicas das formulações. Essas curvas indicam a viscosidade, ou resistência de um fluido ao escoamento, mostrando a força necessária
TABELA 3 - Percentuais (\%) de adequação de vitaminas em relação à ingesta diária recomendada para adultos [3] em 2 L de dieta.

\begin{tabular}{lrrr}
\hline \multicolumn{3}{c}{ Vitaminas } & \multicolumn{2}{c}{ FCM $^{1}$} & \multicolumn{1}{c}{ F1 $^{2}$} & \multicolumn{1}{c}{ F2 $^{2}$} \\
\hline Retinol equivalente & 194,24 & 176,66 & 143,54 \\
Tiamina & 34,66 & 85,00 & 81,43 \\
Riboflavina & 36,00 & 86,75 & 77,38 \\
Niacina & 114,98 & 111,50 & 111,22 \\
C & 18,67 & 54,83 & 54,83 \\
D & 00,00 & 185,50 & 168,00 \\
E & 14,16 & 69,71 & 64,16 \\
K & 304,50 & 233,63 & 233,63 \\
B12 & 696,00 & 595,00 & 470,00 \\
B6 & 82,53 & 74,00 & 284,00 \\
Ácido fólico & 36,80 & 94,45 & 81,95 \\
\hline${ }^{1}$ FCM modificada [5]; ${ }^{2}$ F1 $^{2}$ F2 formuladas no NEPA.
\end{tabular}

TABELA 4 - Percentuais (\%) de adequação de minerais em relação à ingesta diária recomendada para adultos [3] em 2 L de dieta.

\begin{tabular}{lrrr}
\hline & \multicolumn{2}{c}{ Formulações } & \multicolumn{1}{c}{ F2 $^{2}$} \\
\hline Minerais & FCM ${ }^{\mathbf{2}}$ & F1 $^{2}$ & 98,07 \\
Fólcio & 12,93 & 101,56 & 126,15 \\
Ferroro & 107,60 & 140,03 & 135,67 \\
Magnésio & 97,51 & 147,09 & 78,03 \\
Zinco & 36,33 & 97,07 & 105,67 \\
Cobre & 115,29 & 110,00 & 82,80 \\
lodo & 31,44 & 84,10 & 138,48 \\
Selênio & 26,05 & 135,48 & 9,29 \\
Manganês & 32,63 & 17,14 & 36,60 \\
\hline
\end{tabular}

${ }^{1} \mathrm{FCM}$ modificada [5]; e ${ }^{2} \mathrm{~F} 1$ e ${ }^{2} \mathrm{~F} 2$ formuladas no NEPA.

para o fluido escoar numa determinada vazão, dadas, respectivamente, pela tensão de cisalhamento e pela taxa de deformação. A chamada viscosidade aparente (em Pa.s) é calculada pela razão entre a tensão de cisalhamento (em Pa) e a taxa de deformação obtida a partir dessa tensão (em $1 / \mathrm{s}$ ), ou seja, a viscosidade aparente a uma dada taxa de deformação (ou vazão) é representada pela secante à curva por este ponto e pela origem. As curvas mostram que a viscosidade aparente das amostras não é constante e que as formulações F1 e F2 possuem viscosidades menores que a fórmula FCM.

\section{4 - CONCLUSÃo}

Comparando-se as fórmulas F1 e F2 com a fórmula FCM, podemos concluir que foi possível obter formulações normoprotéicas, normolipídicas e normoglicídicas, com maiores teores de fibra alimentar, densidade padrão de $1 \mathrm{kcal} / \mathrm{mL}$ e menor viscosidade, utilizando-se alimentos convencionais de baixo custo, fácil aquisição e factíveis de preparo em domicílio.

A adição de fubá de milho, Soymilk ${ }^{\circledR}$ e Nidex $^{\circledR}$ na composição das fórmulas melhorou o valor nutritivo e a fluidez das soluções, viabilizando administração de maiores quantidades de nutrientes em menos tempo, com menor exposição da dieta à temperatura ambiente. 


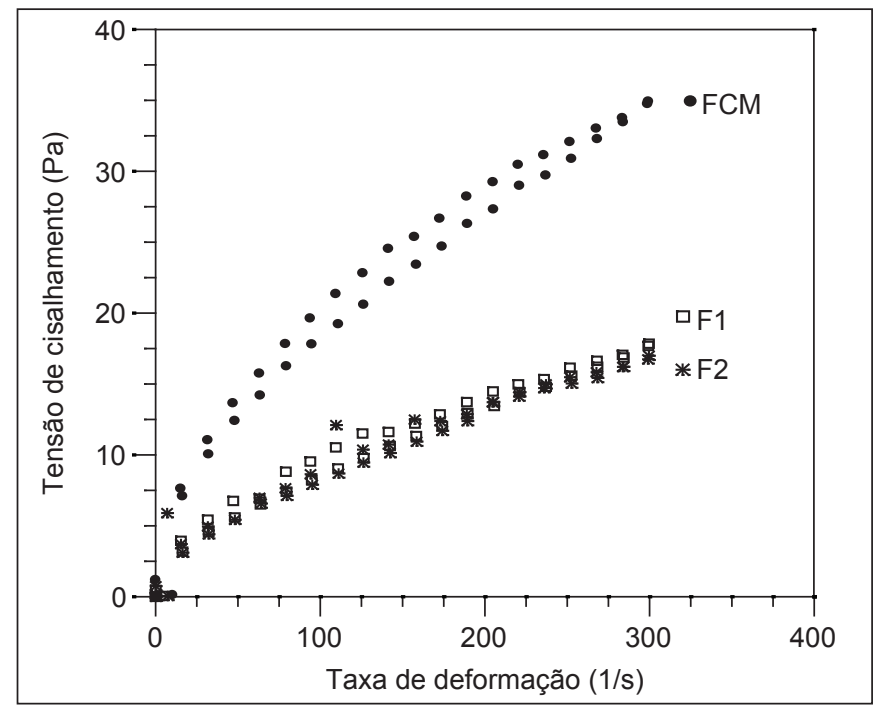

FIGURA 2 - Viscosidade aparente das formulações FCM, F1 e F2 a $23{ }^{\circ} \mathrm{C}$.

As formulações F1 e F2 podem ser opções para uma individualização de dieta normal oral ou enteral por gastrostomia em terapia domiciliar.

A fórmula F2 apresentou valor nutritivo semelhante ao da F1, porém com teor mais baixo de colesterol.

Modificações nas proporções dos alimentos (menor quantidade de carne) na formulação FCM devem ser feitas para se obter solução mais fluida. Não é recomendado acondicionamento dessa dieta em refrigerador para evitar aumento da viscosidade desta, sendo preferível o uso logo após o preparo.

\section{5 - REFERÊNCIAS BIBLIOGRÁFICAS}

[1] BAXTER, Y. C.; WAITZBER, D. L.; RODRIGUES, J. J. G.; PINOTTI, H. W. Critérios de decisão na seleção de dietas enterais. In: WAITZBERG, D. L (Ed). Nutrição oral, enteral e parenteral na prática clínica. $3^{\mathrm{a}} \mathrm{ed}$. São Paulo: Atheneu, 2000. Cap. 41, p. 659-676.

[2] BAXTER, Y. C.; CECCONELO, I. Nutrição enteral domiciliar. In: PINOTTI, H. W. Nutrição enteral em cirurgia. São Paulo: Fundo Editorial BYK, 1997. p. 198-201.

[3] BRASIL. Portaria n. 33 de 13 de janeiro de 1998. Ingesta diária recomendada para adultos. Ministério da Saúde - Secretaria de Vigilância Sanitária. Brasília, DF. Disponível em: <http://www.anvisa.gov.br/alimentos/legis/especifica/regutec.htm>. Acesso em: 5 abr 2004.

[4] CARPER, J. Total nutrition guide. New York: Bantam Books, 1989.

[5] CARVAlHO, E. B. A nutrição no contexto médico. Rev. Bras. Nutr. Clin., São Paulo, v. 12, n. 4, p. 135-138, 1997.

[6] CARVAlho, E. B.; COUTO, C. M. F.; SAlES, T. R. A. Manual de suporte nutricional. Rio de Janeiro: Médica e Científica Ltda., 1992.
[7] CAVALCANTI, M. L. F. Tabela: teor de fibras alimentares. Fibras News. São Paulo: (ed. especial), 1990. p. 8.

[8] COSTA, H. M. C.; LUZ, M. O. R.; CARMONA, M. J. C.; CARDOSO, E.; ISOSAKI, M.; JÚNIOR, J. O. C. A. Reintrodução da alimentação oral em pacientes traqueostomizados com terapia de nutrição enteral. Rev. Bras. Nutr. Clin., São Paulo, v. 18, n. 4, p. 168-172, 2003.

[9] FRANCO, G. Tabela de composição química dos alimentos. 9a ed. São Paulo: Atheneu, 1992.

[10] FUNDAÇÃO INSTITUTO BRASILEIRO DE GEOGRÁFIA E ESTATÍSTICA. Estudo nacional de despesa familiar. Tabela de composição de alimentos. $4^{\text {a }}$ ed. Rio de Janeiro: IBGE, 1977.

[11] HEIMBURGER, D.; WENINSIER, R. L. Guidelines for evaluating and categorizing enteral feeding formulas according to therapeutic equivalence. J. Parent. Ent. Nutr., Baltimore, v. 9, n. 1, p. 61-67, 1985.

[12] HOLLAND, B.; WELCH, A. A; UNWIN, I.; BUSS, D. H; PAUL, A. A; SOUTHGATE, D. A. T. The composition of foods. $5^{\text {th }}$ ed., Cambridge, UK, Richard Clay Ltda, 1992.

[13] KENNEDY, E.; MEYERS, L.; LAYDEN, W. The dietary guidelines for americans: an overview. J. Am. Diet. Assoc., Ilinois, v. 96, n. 3, p. 234-237, 1996.

[14] LII, C.; SHAO, Y.; TSENG, K. Gelation Mechanism and rheological properties of rice starch. Cereal Chem. Taipei, v. 72, n. 4, p. 393-400, 1995.

[15] LUZ, M. L. G. S; TREPTOW, R. O. Comportamento de variedades tailandesas de arroz. Rev. Bras. Agroc. Pelotas, v. 4, n. 3, p. 151-157, 1998.

[16] MAIA, L. H.; WANG, S. H.; ASCHERI, L. C.; CABRAL, M. C.; FERNANDES, M. S. Viscosidade de pasta, absorção da água e índice de solubilidade em água dos mingaus desidratados de arroz e soja. Rev. Ciênc. Tecnol. Aliment. Campinas, v. 19, n. 3, p. 1-13, 1999.

[17] MARLETT, J. A; SLAVIN, J. L. Position of the American dietetic association: health implications of dietary fiber. J. Am. Diet. Assoc. Ilinois [online], v. 97, n. 10, p. 1157-1163, 1997. Disponível em: <http:/www.feberwater.com.index4.html>. Acesso em: 1 abr 2004.

[18] MITNE, C.; SIMÕES, A. M. G.; WAKAMOTO, D.; LIORI, G. P.; SULLIVAN, M.; COMER, G. M. Análise das dietas enterais artesanais. Rev. Bras. Nutr. Clín., São Paulo, v. 16, n. 3, p. 100-109, 2001.

[19] MITNE, C. Preparações não-industrializadas para nutrição enteral. In: WAITZBERG, D. L (Ed). Nutrição oral, enteral e parenteral na prática clínica. $3^{\mathrm{a}} \mathrm{ed}$. São Paulo: Atheneu, 2000. Cap. 39, p. 629-640.

[20] PENnington, J. A. T. Dietary nutrient guide. California: The Avi Publishing Company INC, 1976.

[21] SCHNEIDER, S. M.; RAINA, C.; PUGLIESE, P.; POUGE, I.; RAMPAL, P.; HÉBUTERNE, X. Outcome of patients treated with home enteral nutrition. J. Parent. Ent. Nutr., Baltimore, v. 25, n. 4, p. 203-209, 2001.

[22] SGARBIERI, V. C. Proteínas em alimentos protéicos. São Paulo: Livraria Varela, 1996.

[23] SHAW, D. J. Introdução à química dos colóides e de superfícies. São Paulo: Edgard Blucher, 1975.

[24] SILK, D. B. A. Diet formulation and choice of enteral diet. Gut., London, v. 27, n. Sl, p. 40-46, 1986. 
[25] STEPHAN, A. B.; BRITO, S.; MONTONE, M. Perfil nutricional dos pacientes com terapia nutricional enteral em assistência domiciliária. Rev. Bras. Home Care, Rio de Janeiro, n. 93, p. 26-28, 2003.

[26] WAITZBERG, D. L. Fibras em nutrição enteral. Newsletter Nutrição enteral XLII. Jan 2004; Disponível em: < http://www.nutritotal.com.br > . Acesso em: $5 \mathrm{abr}$ 2004.

[27] WAITZBERG, D. L. Nutrição enteral e parenteral na prática clínica. $2^{\text {a }}$ ed. São Paulo: Atheneu, 1995.
[28] WAITZBERG, D. L et al. Eficácia e tolerância de uma nova formulação dietética enteral em doentes desnutridos. Rev. Ass. Med. Bras., São Paulo, v. 31, n. 11/12, 1985.

[29] WORLD HEALTH ORGANIZATION (WHO). Diet, nutrition and the prevention of chronic diseases, a report of the World Health Organization study group on diet, nutrition and prevention of noncommunicable diseases. Nutr. Reviews, Boston, v. 49, n. 10, p. 291-301, 1991. 